\title{
Giant Electrorheological Effect: A Microscopic Mechanism
}

\author{
Shuyu Chen, ${ }^{1}$ Xianxiang Huang, ${ }^{1}$ Nico F. A. van der Vegt, ${ }^{2}$ Weijia Wen, ${ }^{1}$ and Ping Sheng ${ }^{1, *}$ \\ ${ }^{1}$ Department of Physics and William Mong Institute of Nano Science and Technology, \\ Hong Kong University of Science and Technology, Clear Water Bay, Kowloon, Hong Kong, China \\ ${ }^{2}$ Center of Smart Interfaces, Technical University of Darmstadt, Petersenstrasse 32, 64287 Darmstadt, Germany
}

(Received 5 November 2009; published 19 July 2010)

\begin{abstract}
Electrorheological fluids constitute a type of colloids that can vary their rheological characteristics upon the application of an electric field. The recently discovered giant electrorheological (GER) effect breaks the upper bound of the traditional ER effect, but a microscopic explanation is still lacking. By using molecular dynamics to simulate the urea-silicone oil mixture trapped in a nanocontact between two polarizable particles, we demonstrate that the electric field can induce the formation of aligned (urea) dipolar filaments that bridge the two boundaries of the nanoscale confinement. This phenomenon is explainable on the basis of a 3D to 1D crossover in urea molecules' microgeometry, realized through the confinement effect provided by the oil chains. The resulting electrical energy density yields an excellent account of the observed GER yield stress variation as a function of the electric field.
\end{abstract}

DOI: 10.1103/PhysRevLett.105.046001

PACS numbers: $83.80 . \mathrm{Gv}, 83.60 . \mathrm{Np}$

Electrorheological (ER) fluids [1-15] are a type of colloidal dispersions which can vary their rheological characteristics through the application of an external electric field. The traditional ER mechanism is based on induced polarizations arising from the dielectric constant contrast between the solid particles and the fluid $[6,12]$. The recent discovery of the giant electrorheological (GER) effect [712], in urea-coated barium titanyl-oxalate nanoparticles $\left[\mathrm{NH}_{2} \mathrm{CONH}_{2} @ \mathrm{BaTiO}\left(\mathrm{C}_{2} \mathrm{O}_{4}\right)_{2}\right]$, or BTRU for short, dispersed in silicone oil, has shown that the theoretical upper bound of the ER effect is no longer applicable to this new type of materials. Instead, a phenomenological model of the GER mechanism, based on aligned urea molecular dipoles in the small contact regions of the nanoparticles, yielded an adequate account of the observed effect $[7,9,12]$. However, a microscopic picture of how this can occur has so far eluded persistent efforts. Moreover, as the GER effect is highly sensitive to whether the dispersing oil can wet the solid particles $[10,11]$, in contrast to the traditional ER fluids, a natural question is how this observation can be integrated into a coherent GER mechanism. In view of the fact that the GER effect has now been reproduced in many different material systems and therefore is becoming a much more general effect $[14,15]$, answers to the above questions would not only be timely, but may also shed light on how to devise general strategies for harnessing and controlling the large electric energy stored in molecular dipoles.

In this work we use molecular dynamics (MD) simulations to show that in a mixture of urea molecules with silicone oil chains confined between two bounding surfaces (denoted as substrates below) of a nanoscale contact, aligned urea molecular dipoles can form filaments snaking through the pores of the oil film to bridge the substrates. The required electric field for aligning the urea dipoles is found to be lowered by a factor of 2 to 3 in the presence of the oil chains, compared to that without the oil chains. Moreover, the formation of aligned dipolar filaments is an interfacial phenomenon, which directly implies the surface area scaling characteristic of the GER mechanism [8] that favors nanoparticle dispersions. A nonwetting oil would give rise to phase separation and the formation of large aggregates, thus suppressing the GER effect $[10,11]$. We give a simple argument, involving the dimensionality dependence of the polarizability for molecular dipoles, to explain all the observed phenomena in the simulations. Calculated electrical energy density shows that the aligned dipolar filaments can give rise to very significant attractive interaction between the confinement substrates. Data sets for the GER yield stress are shown to exhibit scaling behavior explainable by our simulation results with reasonable parameter values. Furthermore, the mechanism of molecular dipole alignment under 1D confinement shows that the GER effect could be only one aspect of its manifested consequences. Formation of water filaments under constrained conditions may give rise to forces far stronger and longer in range than the van der Waals interaction. We give a plausible speculation on the fluctuation-induced molecular dipole alignment at the end of this Letter.

Owing to the nonideal thermodynamic nature of the urea-water solution, it is known that urea molecules tend to predominantly aggregate at the surface of hydrophilic solid particles [16,17], forming a (nonuniform) liquidlike coating [9]. Accordingly, we simulate our system of nanoparticles' contacts by two parallel bounding substrates, separated by a $2.9 \mathrm{~nm}$ gap. Molecular dynamics simulations were performed with the package GROMACS [18], using parameterized force fields to describe urea [19], polydimethylsiloxane (silicone oil) [20], and water [21] molecules contained in a rectangular box with periodic 
boundaries. Force fields in the simulations arise from bonding interactions and nònbonding interactions, i.e., the van der Waals and electrostatic interactions. Bond lengths were constrained using the SHAKE [22] method, with a time step for integration of 2 fs. Long-range Coulomb interaction was calculated with the particle mesh Ewald algorithm [23], using tin-foil boundary conditions, a grid spacing of $0.12 \mathrm{~nm}$ and a real-space cutoff of $1.1 \mathrm{~nm}$. The van der Waals forces are cut off at the same distance, appropriate for the Lennard-Jones interaction potential. More details about the force fields are given in [24]; their relevant parameter values have been widely used and scrutinized [24]. It should be noted that the coupling to the heat bath is accomplished via the Berendsen algorithm [25].

Silicone oil (polydimethylsiloxane or PDMS) is considered to be part of the system, and we used methyl-group terminated silicone oil chains with 10 monomer units per chain. Silicone oil is a typical dispersant for the GER nanoparticles, with previous experimental studies showing the interaction between the nanoparticles to be dispersant dependent [11]. This indicates that the silicone oil chains have a crucial effect on the GER phenomenon. The fact that the silicone oil wets the particle is attributed to the presence of the oxalate groups in the core nanoparticles, and the nonuniformity of the urea coating. In this simulation we consider only a sandwichlike urea-silicone oil-urea mixture confined in the gap region, with one layer of water molecules at each boundary where they are each restrained to a configuration in equilibrium by a harmonic potential [26]. Both the presence of water as well as the "soft" restraining potential is physically reasonable as it is known that each barium titanyl-oxalate molecule carries ten water molecules, with seven of them associated with barium atoms and the remaining three occupying interstitial sites [27]. An equilibrium run was first performed at $298 \mathrm{~K}$ with no applied field. Because of the hydrophilic nature of urea and the hydrophobic nature of silicone oil, urea molecules were initially separated by a silicone oil film in the gap.

When the electric field was turned on, the urea molecules were seen to diffuse into the silicone oil layer from both sides, with their molecular dipoles generally aligned along the field direction, forming filaments that bridge the two sides of the gap. Figure 1(a) shows a snapshot taken from the simulation to illustrate the configuration of the system under a $0.2 \mathrm{~V} / \mathrm{nm}$ electric field. The filament structure may be clearly discerned in the left panel of Fig. 1(a). In the right panel we explicitly delineate the hydrogen bonds between the urea molecules in the filaments. A typical dipole moment in the filament is $>3 \mathrm{D}$ along the direction of the electric field. The formation of the filaments always starts at the substrates, and no formation of filaments was ever observed in our simulations for gaps $>10 \mathrm{~nm}$. Thus the formation of filaments (and therefore the enhanced alignment) is inherently an interfacial effect a

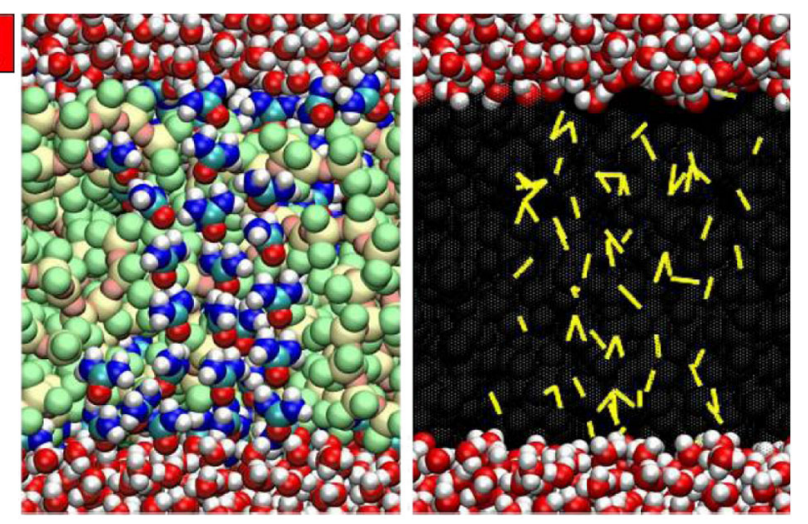

b

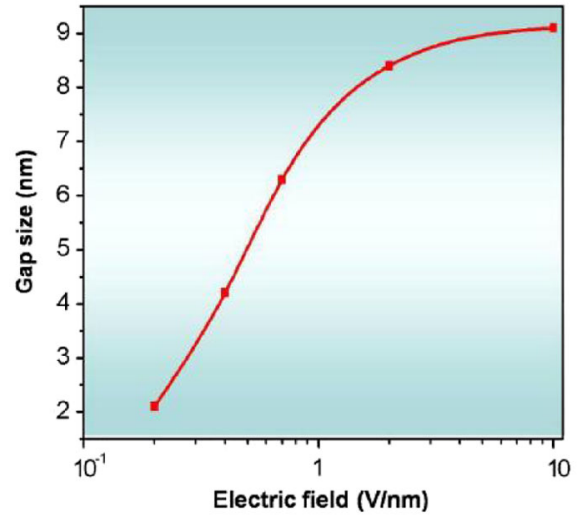

FIG. 1 (color online). (a) Snapshot taken from one of the MD runs which shows the urea filament structure under a $0.2 \mathrm{~V} / \mathrm{nm}$ electrical field. The gap is $2.9 \mathrm{~nm}$ in this case. The methyl group is represented by a single green (medium-light gray) sphere. Oxygen, hydrogen, nitrogen, carbon, and silicon atoms are denoted by red (medium gray), white, blue (medium-dark gray), navy blue (dark gray), and yellow (light gray), respectively. Only part of the water boundary layer (red [medium gray]-white) is shown. The direction of the field is perpendicular to the boundary layers. In the right panel, we show the hydrogen bondings (highlighted by bright yellow lines) between the urea molecules in the filament structure. Here the silicone oil chains are darkened in order to delineate the hydrogen bonds. (b) Maximum thickness of the oil layer in the gap plotted as a function of applied electric field. Solid line is a guide to the eye.

with a decay length $<4-5 \mathrm{~nm}$. Figure 1(b) shows the results of varying the gap size and finding the field at which the filaments are no longer observed. It is seen that for large fields, the gap size saturates at $8-9 \mathrm{~nm}$. This essentially sets a length scale for the GER effect. Owing to the small magnitude of this length scale, the GER effect may be considered an interfacial phenomenon, with its magnitude scaling [8] with the interfacial area.

The electric field required to align the molecular dipoles may be defined by the point where the average $|-\vec{p} \cdot \vec{E}|$ energy becomes linear in $E$. It is found that in the present case the threshold field is $\sim 0.2 \mathrm{~V} / \mathrm{nm}$. We have also examined the case in which the oil chains are absent, replaced by a homogeneous dispersion of urea molecules with a density set at $1.32 \mathrm{~g} / \mathrm{cm}^{3}$. The alignment field in 
that case is $\sim 0.5 \mathrm{~V} / \mathrm{nm}$. Thus the presence of oil lowers the aligning field by a factor of $\sim 2.5$ [24].

The formation of filaments, with the attendant lowering of the aligning field and the finite penetration length, may be attributed to the confinement effect exerted by the oil chains. Because of the hydrophobic nature of the oil chains, there are repulsive interactions between oil chains' methyl groups and atoms in the urea molecules that can act as either a donor or an acceptor when forming hydrogen bonds. Such repulsive interactions facilitate the formation of a single urea molecular file, consisting of multiple urea filaments confined to the "axes" of the hydrophobic pore space that is forced open by the urea dipolar file (left panel of Fig. 1(a)]. Thus the confinement, which tends to decrease the entropic phase space of the urea dipoles, works in concert with the hydrogen bonding interactions [right panel of Fig. 1(a)] to significantly increase urea dipoles' sensitivity to the applied field. That is, for 3D the thermally-averaged dipole moment along the field direction is given by the Langevin function $\langle p\rangle_{3 \mathrm{D}} / p_{0}=$ $\operatorname{coth}\left(p_{0} E / k_{B} T\right)-\left(k_{B} T / p_{0} E\right)$, where for urea molecules $p_{0}=4.6 \mathrm{D}$ [28]. But for $1 \mathrm{D}$, it is given by $\langle p\rangle_{1 \mathrm{D}} / p_{0}=$ $\tanh \left(p_{0} E / k_{B} T\right)$ [24]. At any given $E, \Delta p=\langle p\rangle_{1 \mathrm{D}}-\langle p\rangle_{3 \mathrm{D}}$ is always positive (e.g., at $0.3 \mathrm{~V} / \mathrm{nm}$ it is $2.12 \mathrm{D}$ ); therefore $-\Delta p E$ provides a driving force for the urea molecules to develop a more diffuse interface with the oil film, in the form of the 1D filaments' penetration. The width of the diffuse urea-oil interface should reflect the magnitude of the driving force. The presence of hydrogen bonds between the urea molecules in the filaments means that their energetic contributions (per urea molecule) can help to counterbalance those in the (3D) urea molecular dispersion. The electrical alignment energy thereby emerges as the dominant factor. We note that for $\langle p\rangle_{1 \mathrm{D}}=\langle p\rangle_{3 \mathrm{D}}=0.8 p_{0}$, the required electric field value for the $1 \mathrm{D}$ case is only 0.25 times that of the 3D case. Thus the alignment field is considerably reduced by the dimensionality effect, just as observed in simulations. Moreover, as $E \rightarrow \infty$, we have $\Delta p \rightarrow k_{B} T / E$, which implies $-\Delta p E$ approaches a constant value, $-k_{B} T$, independent of $E$. This is consistent with saturation behavior as seen in Fig. 1(b). A more quantitative version of the theory will be reported elsewhere. We note that molecular filament formation has been observed in previous MD simulations [29,30] under a different context.

The contact region between two nanoparticles is responsible for much of the overall rheological characteristics of the nanoparticle suspensions, and this is especially true for the GER effect, in which the suspension can become solidlike under an applied electric field, with an attendant yield stress. To obtain the relation between the yield stress and the electric field, we calculate the total potential energy density of the simulation box at different fields, with the zero-field total potential energy subtracted off. The resulting difference $\Delta E$ is negative, indicating a large attraction between the two bounding surfaces. Plotted in Fig. 2 is $\Delta W$, given by $\Delta E$ divided by the volume of our simulation sample, $190 \mathrm{~nm}^{3}$, as a function of the electric field (red curve). Since the yield stress is directly proportional to $\Delta W$ as seen below, all the experimental yield stress vs electric field data should be able to be scaled onto this relation, if the simulated effect is indeed the GER mechanism.

The electric field at the contact region of two nanoparticles is enhanced by a factor of $\alpha \sim 100-300$ (compared to the experimentally applied electric field) [31], owing to the large dielectric constant of the nanoparticles and hence the field concentration effect at the contact region $[9,14,15]$. Thus $0.5 \mathrm{~V} / \mathrm{nm}$ field in the gap region would correspond roughly to $\sim 5000 \mathrm{~V} / \mathrm{mm}$ (or less) of experimentally applied field. From Fig. 2, we obtain $\sim 25 \mathrm{MPa}$ to be the energy density at $0.5 \mathrm{~V} / \mathrm{nm}$. This energy density must be scaled by a volume dilution factor $\beta$ for the energy density of the GER fluid, since the nanoscale gap considered here constitutes the region of closest approach between two nanoparticles. Hence the gap electrical energy should be averaged over a volume on the order of $d^{3}, d$ being the nanoparticle diameter. For $d \sim 50-100 \mathrm{~nm}$ and our sample volume of $190 \mathrm{~nm}^{3}$, $\beta \sim 1000-10,000$. Both $\alpha$ and $\beta$ are potentially measur-

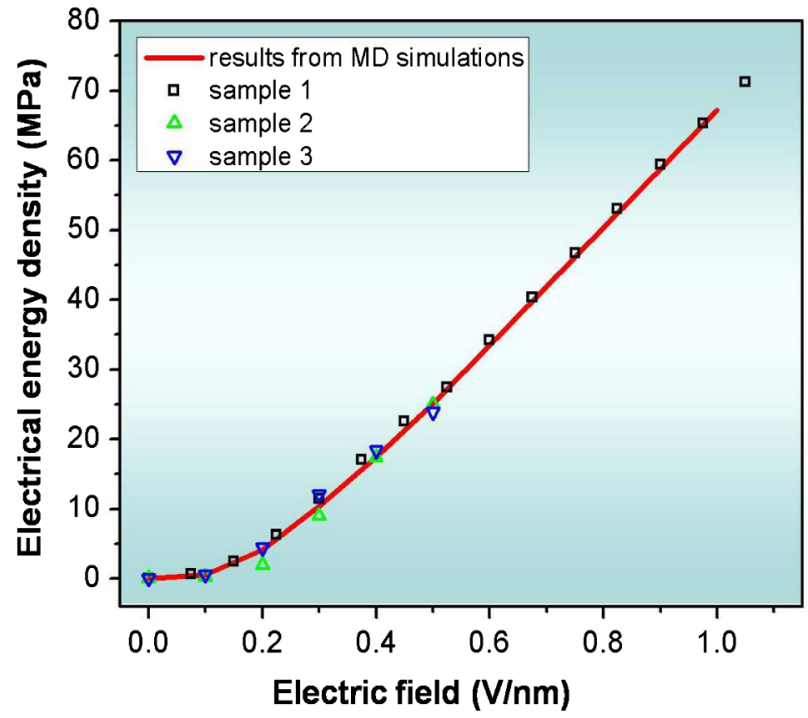

FIG. 2 (color online). Electrical energy density plotted as a function of applied electric field. Scaled data from three sets of experiments are superposed on the curve. The electric field $E$ is obtained from the measured field $E_{m}$ by $E=\alpha E_{m}$, where $\alpha$ is the field enhancement factor (see text for details). The measured yield stress $Y$ is related to the electrical energy density $\Delta W$ by $\Delta W=\beta Y / 10$, where $\beta$ is the volume dilution factor. Sample 1 (Ref. [7]) is a suspension comprising 50-70 $\mathrm{nm}$ nanoparticles dispersed in silicone oil; Sample 2 (Ref. [8]) is composed of smaller particles; Sample 3 (Ref. [11]) has slightly larger particles dispersed in hydroxyl-group-terminated silicone oil. The values of $(\alpha, \beta)$ for samples 1,2 and 3 are (281.2 ,7590), (100, 974), (100, 1010), respectively. 
able by using an atomic force microscope (AFM) with appropriate setup [31].

The stress may be expressed as $\tau=(1 / \beta)[\partial(\Delta W) / \partial \varepsilon]$, where $\varepsilon$ denotes the strain. For a linear stress-strain relation $\tau=a \varepsilon$, it follows that $\Delta W=(\beta / 2) a \varepsilon^{2}$. Here the factor $\frac{1}{2}$ may be replaced by a larger factor if the stressstrain relation deviates from linearity at large strain values, but such deviations can be absorbed into the $\beta$ factor. Since yield stress $Y=\tau_{y}=a \varepsilon_{0}$, we have $Y=\tau_{y}=2 \Delta \mathrm{W} / \beta \varepsilon_{0}$, where $\varepsilon_{0}=0.2$ (radian) is the strain at the yield point [7]. By suitably scaling the experimental data by $\alpha$ and $\beta$ values as indicated in the caption to Fig. 2 , it is seen that all three data sets fall on the curve obtained from the MD simulations. As the values of $\alpha$ and $\beta$ fall within the physically reasonable range, our simulation results thus offer a microscopic account of the GER effect.

A potentially new form of interaction between two polarizable substrates may arise from the relatively low molecular dipole alignment field of $0.2 \mathrm{~V} / \mathrm{nm}$. Consider the nanoscale gap as a small capacitor in thermal bath which would have $k_{B} T / 2$ of thermal energy in accordance with the equipartition principle $[32,33]$. By equating this thermal energy to $C\left\langle V^{2}\right\rangle / 2$, where $C$ denotes the capacitance [34] and $\langle\cdots\rangle$ denotes thermal averaging, it is easy to obtain $\sqrt{\left\langle V^{2}\right\rangle}=\sqrt{k_{B} T / C} \sim 0.13 \mathrm{~V}$ for the system considered in this work, or a field $\sim 0.05 \mathrm{~V} / \mathrm{nm}$. Such an average field should already be able to partially align the permanent dipoles to some degree, especially if the gap is smaller than $2 \mathrm{~nm}$. Moreover, the fluctuation effects can be enhanced for substrates that have asperities, leading to reduced local capacitance (therefore large local fields) and hence attractive forces on the two bounding surfaces that are much stronger and longer range than that from the van der Waals interaction.

This work is supported by research Grants No. RGC602207 and No. RGC621006. S.C., X.H., and N. vdV. are grateful to Max Planck Institute for Polymer Research where part of the work was performed.

*Corresponding author: sheng@ust.hk

[1] M. Whittle and W. Bullough, Nature (London) 358, 373 (1992).

[2] T. Halsey, Science 258, 761 (1992).

[3] R. Tao and J. M. Sun, Phys. Rev. Lett. 67, 398 (1991).

[4] H. Ma, W. Wen, W. Y. Tam, and P. Sheng, Phys. Rev. Lett. 77, 2499 (1996).

[5] W. Y. Tam et al., Phys. Rev. Lett. 78, 2987 (1997).

[6] H. Ma, W. Wen, W. Y. Tam, and P. Sheng, Adv. Phys. 52, 343 (2003).

[7] W. Wen, X. Huang, S. Yang, K. Lu, and P. Sheng, Nature Mater. 2, 727 (2003).

[8] W. Wen, X. Huang, and P. Sheng, Appl. Phys. Lett. 85, 299 (2004)
[9] X. Huang, W. Wen, S. Yang, and P. Sheng, Solid State Commun. 139, 581 (2006).

[10] C. Shen, W. Wen, S. Yang, and P. Sheng, J. Appl. Phys. 99, 106104 (2006).

[11] X. Gong et al., Nanotechnology 19, 165602 (2008).

[12] W. Wen, X. Huang, and P. Sheng, Soft Matter 4, 200 (2008).

[13] J. Zhang et al., Phys. Rev. Lett. 101, 194503 (2008).

[14] P. Tan et al., J. Phys. Chem. B 113, 9092 (2009).

[15] R. Shen et al., Adv. Mater. 21, 4631 (2009).

[16] A. Wallqvist, D. G. Covell, and D. Thirumalai, J. Am. Chem. Soc. 120, 427 (1998).

[17] L. Hua, R. Zhou, D. Thirumalai, and B. J. Berne, Proc. Natl. Acad. Sci. U.S.A. 105, 16928 (2008).

[18] E. Lindhal, B. Hess, and D. van der Spoel, J. Mol. Mod. 7, 306 (2001).

[19] S. Weerasinghe and P.E. Smith, J. Phys. Chem. B 107, 3891 (2003).

[20] R. M. Sok, H. J. C. Berendsen, and W.F. van Gunsteren, J. Chem. Phys. 96, 4699 (1992).

[21] H. J.C. Berendsen, J. R. Grigera, and T.P. Straatsma, J. Phys. Chem. 91, 6269 (1987).

[22] J.P. Ryckaert, G. Ciccotti, and H. J.C. Berendsen, J. Comput. Phys. 23, 327 (1977).

[23] U. Essman et al., J. Chem. Phys. 103, 8577 (1995).

[24] See supplementary material at http://link.aps.org/ supplemental/10.1103/PhysRevLett.105.046001.

[25] H. J. C. Berendsen et al., J. Chem. Phys. 81, 3684 (1984).

[26] The force constant is chosen to be such so that the positional fluctuations of the boundary water molecules are on the order of its molecular dimension.

[27] W.E. Rhine, R. B. Hallock, W. M. Davis, and W. WongNg, Chem. Mater. 4, 1208 (1992).

[28] Handbook of Chemistry and Physics, edited by R.C. Weast and M. J. Astle (CRC, Boca Raton, 1981), 62nd ed..

[29] G. Hummer, J.C. Rasaiah, and J.P. Noworyta, Nature (London) 414, 188 (2001).

[30] R. Wang, J. Li, H. Lu, and H. Fang, J. Am. Chem. Soc. 127, 7166 (2005).

[31] $\alpha$ and $\beta$ might be obtained experimentally by, for example, an AFM measurement of nanoparticle-nanoparticle interaction in which the AFM tip forms one bounding surface (of known size) so that the microscopic electric field and friction (i.e., shear stress) measured in a nanoscale layer of oil-BTRU mixture bounded by a conducting substrate on the other side can be compared to their macroscopic counterparts. By knowing the particle size of the BTRU particles, the ratios $\alpha$ and $\beta$ may potentially be deduced.

[32] P. Sheng, E. K. Sichel, and J. I. Gittleman, Phys. Rev. Lett. 40, 1197 (1978).

[33] P. Sheng, Phys. Rev. B 21, 2180 (1980).

[34] $C$ is estimated as $\varepsilon_{0} S / d$, where $\varepsilon_{0}$ is the vacuum permittivity, $S=\pi r_{0}^{2}$ is the contact area between two nanoparticles with $r_{0} \approx 5 \mathrm{~nm}$ the equilibrium contact radius at $1 \mathrm{kV} / \mathrm{mm}$ external field (see Ref. [9]), and $d=2.9 \mathrm{~nm}$ is the gap dimension. The estimated value of $C$ is $2.4 \times$ $10^{-19} \mathrm{~F}$. 